\title{
WHATSAPP VS. CAMPUS VIRTUAL INSTITUCIONAL EN TIEMPOS DE COVID-19. PERCEPCIÓN DE LOS ESTUDIANTES DE COMUNICACIÓN SOCIAL
}

\section{WHATSAPP VS. INSTITUTIONAL VIRTUAL CAMPUS IN TIMES OF COVID-19. PERCEPTION OF SOCIAL COMMUNICATION STUDENTS}

Gladys Adriana Espinel Rubio'

\section{Cesar Augusto Hernández Suarez²}

\author{
Raúl Prada Núñez ${ }^{3}$ \\ Universidad Francisco de Paula Santander \\ Colombia
}

\section{RESUMEN}

La pandemia mundial de Covid-19 ha obligado a las instituciones de educación superior a trasladar la educación presencial a la educación remota. Pese a que las universidades tenían acceso a plataformas virtuales para facilitar el proceso enseñanza, aprendizaje y evaluación entre los estudiantes y los docentes, su uso era limitado. Esta investigación examina empíricamente hasta qué punto los docentes

1 Magíster. Universidad Francisco de Paula Santander, Colombia, gladysespinel@ufps.edu.co, ORCID: https://orcid.org/0000-0002-8796-9257

2 Magíster. Universidad Francisco de Paula Santander, Cúcuta. cesaraugusto@ufps.edu.co, ORCID: https:// orcid.org/0000-0002-7974-5560

3 Universidad Francisco de Paula Santander, Cúcuta.raulprada@ufps.edu.co, ORCID: https://orcid. org/0000-0001-6145-1786

usan las plataformas institucionales y por otro lado adaptan la red social WhatsApp para mantener la comunicación académica, desde la perspectiva de los estudiantes. Para lograrlo, se aplicó un cuestionario en línea a 606 jóvenes de cinco programas de Comunicación Social en Colombia. Los resultados mostraron que se ha promovido el uso de WhatsApp para construir comunidadesogruposacadémicos. Sinembargo, las plataformas institucionales se constituyen en el escenario idóneo y legítimo para el desarrollo de las clases. Los comunicadores en formación valoran las competencias tecnológicas y comunicativas de sus profesores, pero desde su percepción, es necesario que se mejoren las pedagógicas en su ejercicio docente mediado 
por TIC. En conclusión, si bien WhatsApp en el ámbito académico podría promover el aprendizaje social y convertirse en un medio para fomentar el aprendizaje en línea, todavía no puede ser asumido como un ambiente virtual de aprendizaje propiamente dicho; los futuros profesionales de la Comunicación requieren de las habilidades y competencias TIC para insertarse en un mercado laboral en donde se impone lo digital y ha sido el confinamiento el escenario para su apropiación desde el aula virtual, así como el aprendizaje por imitación frente al uso que les dan a éstas sus propios docentes.

\section{PALABRAS CLAVES}

Covid-19, comunicación social, digital, universidad, WhatsApp.

\section{ABSTRACT}

The global Covid-19 pandemic has forced face education to remote education. Although universities had access to virtual platforms to facilitate the teaching, learning and assessment process between students and teachers, their use was limited. This research empirically examines the extent to which teachers use institutional platforms and, on the other hand, adapt the WhatsApp social network to maintain academic communication, from the students' perspective. To achieve this, an online questionnaire was applied to 606 young people from five Social Communication programs in Colombia. The results showed that the use of WhatsApp has been promoted to build academic communities or groups. However, institutional platforms constitute the ideal and legitimate setting for the development of classes. Communicators in training value the technological and communication skills of their teachers, but from their perception, it is necessary to improve pedagogical skills in their teaching practice mediated by ICT. In conclusion,

although WhatsApp in the academic field could promote social learning and become a means to promote online learning, it still cannot be assumed as a virtual learning environment itself; Future Communication professionals require ICT skills and competencies to enter a labor market where digital is imposed and confinement has been the setting for its appropriation from the virtual classroom, as well as learning by imitation against the use that they give these their own teachers.

\section{KEYWORDS}

Covid-19, social communication, digital, university, WhatsApp.

\section{INTRODUCCIÓN}

Los sistemas educativos, desde el nivel primario hasta el superior, se han transformado durante el período de aislamiento por la pandemia generada por la crisis de salud del Covid- 19 . En consecuencia, diversas políticas de los gobiernos han cambiado todas las clases presenciales a mediadas por TIC, lo que ha presentado desafíos sin precedentes para la educación presencial.

A pesar de que la mayoría de las universidades cuentan con plataformas tecnológicas y/o sistemas gestión del aprendizaje (LMS) en línea para apoyar el proceso educativo y comunicarse con los estudiantes o con sus docentes, son latentes las debilidades en el proceso enseñanza y aprendizaje pues los implicados no cuentan con todas las capacidades para respaldar el educación en línea. Es claro que por sí misma la tecnología y la plataforma educativa no marcan la diferencia, sino que requieren de la mediación de contenidos por parte de docente formados en los aspectos digitales. (Sanchéz, 2009; VargasCubero \& Villalobos-Torres, 2018; Prada-Núñez et al., 2019). 
En Colombia, las universidades han alentado a sus docentes a utilizar sus plataformas tecnológicas para la gestión del aprendizaje, así como también los sistemas de videoconferencia, por ejemplo, Google Classroom y Zoom. A su vez, los docentes por su cuenta han incorporado el uso de las redes sociales (Facebook y Twitter especialmente) y sistemas de mensajería instantánea para comunicarse con sus estudiantes, sobre todo WhatsApp, por estar incluido sin costo en los planes de datos ofrecidos por los operadores de telefonía móvil.

En Colombia WhatsApp ha dado soporte a la educación primaria y secundaria, especialmente en los estratos socioeconómicos 1,2 y 3 puesto que al no tener consumo de datos celulares permite la conexión continúa, enviar y recibir trabajos escolares, comunicarse, informarse, creargrupos de trabajo y vídeollamadas grupales, facilitando lo entretenido y lo educativo, siendo un sistema que permite el uso y la gratificación inmediata y efectiva (Espinel et al., 2021).

En esta masificación de la comunicación en línea entre los docentes y los estudiantes con fines académicos como consecuencia del Covid-19, las redes sociales se han convertido en una oportunidad para fomentar el aprendizaje en línea con sus estudiantes, especialmente para aquellos que por la falta de conocimiento y habilidades no hacen uso de las plataformas digitales (Espinel-Rubio et al., 2020). Dicha práctica es más común en los niveles precedentes, sin embargo, estudios anteriores sobre el uso de las redes sociales en la educación superior como el de Prada-Núñez et al. (2020) evidencian que se pueden utilizar para apoyar la comunicación con los estudiantes y complementar su aprendizaje.

Sin embargo, esta investigación examina hasta qué punto los docentes y estudiantes de los programas de Comunicación Social en cinco universidades colombianas adoptan el uso académico de la red social WhatsApp y las plataformas digitales institucionales, especialmente después del inicio de la pandemia de Covid-19. Además, analiza aspectos que incluyen la enseñanza y el aprendizaje, el apoyo a los estudiantes, la construcción comunidades en línea entre otros

\section{LAS TIC Y LO DIGITAL EN LOS PROGRAMAS DE COMUNICACIÓN SOCIAL}

Para marzo del 2020, en Colombia había un total de 185.176 estudiantes matriculados en programas universitarios virtuales frente a 1.882.749 en programas presenciales de acuerdo con la Asociación Colombiana de Universidades (ASCUN). La cifra en ambas modalidades descendió en 113 por ciento para el segundo semestre del 2020 , sin que se tengan aún datos consolidados para el 2021.

En el país hay 124 programas de pregrado en Comunicación Social, Periodismo, Comunicación Digital y afines, de los cuales 43 se ofrecian en modalidad a distancia y 81 en modalidad presencial de acuerdo con el Sistema Nacional de Información de la Educación Superior (SNIES). Con la declaratoria de emergencia sanitaria, la oferta presencial migró a la virtualidad mediante encuentros sincrónicos y asincrónicos a través de plataformas como Meet de Google, Teams de Microsoft y Zoom o de las creadas por las mismas universidades para cursos mixtos (B-learning) diseñados antes de la pandemia y soportadas en Moodle y Coursera, entre otros.

Esto significó una dificultad para los docentes y estudiantes que no contaban con dispositivos electrónicos inteligentes que les permitieran soportar dichas plataformas ni con suficiente conectividad pese a que el país contaba con 7,1 millones de accesos fijos a Internet, según datos del Ministerio de las TIC. En un estudio previo, García et al. (2018) hallaron que el 34\% de estudiantes de Comunicación Social en Norte de Santander (una de las regiones abordadas 
en la presente investigación) indicaron no tener acceso a las herramientas, de tal manera que se encuentran limitados en el desarrollo de competencias TIC; pese a esto, se llevó a cabo un proceso apresurado de transformación digital con el consecuente cambio en los modos de enseñar y de aprender.

Es claro que cuando las TIC son consideradas como parte de una estrategia integral de apoyo al establecimiento, pueden mejorar aspectos claves de la cultura escolar, tales como currículo, pedagogía, evaluación, desarrollo profesional de docentes, etc. Este argumento reduce las expectativas de una relación causal entre éstas y el aumento en el logro de aprendizaje de los alumnos, argumentando que éstas sólo facilitan condiciones claves para dicha mejora como lo señaló la OECD hace dos décadas.

En efecto, las TIC juegan un papel determinante en el entramado social, puesto que:

Tienen una especial importancia porque afecta a prácticamente a todos los ámbitos de la actividad de las personas, desde las formas y prácticas de organización social, hasta la manera de comprender el mundo, organizar esa información y transmitirla a otras personas. (Coll \& Monereo, 2008, p.22)

Esta afirmación muestra que las TIC influyen en los aspectos socioculturales del ser humano permitiéndole crear nuevas visiones de mundo a partir de la interactividad que ofrecen estas en el contexto.

En los programas de formación en Comunicación Social las competencias en el uso y apropiación de las TIC son consideradas fundamentales, por cuanto la profesión se ha movido hacia lo digital (USQUID, 2015), incluso en campos que no son exclusivos del ecosistema digital, como el audiovisual (Besalú-Casademont et al., 2017); sin embargo en el país la oferta de pregrado y posgrado con este énfasis todavía es incipiente. De las 273 programas de pregrado y posgrado en Comunicación solo existen 21 ofertas de comunicación digital, comunicación transmedia o multimedia de acuerdo con datos del SNIES.

Por supuesto esta inserción del profesional de la Comunicación en lo digital, no lo eximen de poseer las competencias de pensamiento crítico, dominio de la lengua nativa e idiomas, la creatividad, la capacidad de trabajar en equipo y la organización, "pero surge la necesidad de adquirir nuevas competencias estrechamente relacionadas con el sector digital (competencias multimedia, transmisión de noticias y de producción de información en tiempo real, dominio de las redes sociales, conocimientos de tecnología móvil, interactividad, etc.)" (Ventura et al., 2018, p. 348-349).

En este mismo sentido, laAsociación Colombiana de Facultades y Programas de Comunicación Afacom (2020) concluyó que pese a la demanda porlaformación tecnológica que incluye el manejo de herramientas, plataformas, dispositivos y la comunicación digital, los graduados, empleados y emprendedores insisten en la formación ético-política, la formación sociohumanística y las herramientas teóricas y metodológicas para las realidades sociohistóricas locales y los fenómenos regionales e internacionales.

Otra de las competencias valoradas en los profesionales de la Comunicación Social es la de aprender a aprender; en relación a lo digital, los graduados han tenido que poner a prueba esta capacidad por cuanto los desarrollos tecnológicos varían y evolucionan aceleradamente. Pese a las dificultades en términos de salud mental y economía, la pandemia generó la necesidad de ampliar conocimientos frente al uso de las TIC siendo las aulas virtuales el escenario indicado para retar tanto estudiantes como a docentes en su desempeño en este ámbito. 
Así las cosas, si a los graduados se les exige dichas competencias, los docentes son los llamados a promoverlas, afianzarlas e instaurarlas en su práctica pedagógica.

\section{WHATSAPP COMO HERRAMIENTA EDUCATIVA}

WhatsApp con más de 2000 millones de usuarios en todo el mundo, es la aplicación de mensajería más popular del mundo. Permite enviar y recibir mensajes instantáneos. También posibilita el intercambio de audios, videos y fotografías. Por lo que utilizado como recurso educativo proporciona una serie de ventajas como la compartir información entre los miembros de la clase, creación de grupos de diversos intereses o de debate, coordinar y dar indicaciones sobre una actividad educativa entre otras. Estas posibilidades permiten guiar el aprendizaje, motivar a los estudiantes a participar y fomentar el trabajo colaborativo (Montilla, 2020; AndújarVaca \& Cruz-Martínez, 2017) por lo que a

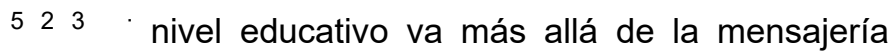
instantánea (Rodríguez, 2020).

Es claro que estas herramientas y aplicaciones, no son generadoras de conocimiento pero ayudan al docente y estudiante a mejorar su comunicación, generar procesos flexibles y abiertos de enseñanza y aprendizaje, además de interesar y motivar (Suárez, 2018).

Sin embargo, Fondevila-Gascón et al. (2019) hallaron en su investigación que los estudiantes universitarios españoles manifestaron una alta dependencia a las estas aplicaciones, incluso, sufren de ansiedad y estrés ocasionados por un uso excesivo, lo que genera problemas de salud y peor rendimiento académico. Cerca del $97 \%$ utiliza la aplicación más de 10 veces al día y el $52,40 \%$ reconoció perder horas de sueño por mantener conversaciones nocturnas.

Se destaca también de dicha investigación que el $50 \%$ sufre del síndrome de "vibración fantasma" y el $37 \%$ revisa su teléfono en caso de no recibir mensajes o llamadas por algún lapso prolongado. Leer o escribir un mensaje de WhatsApp es su primera y última actividad diaria, para el $54,22 \%$ y el $59,04 \%$, respectivamente.

Por su parte, Vilches y Reche (2019), encontraron que el uso de las aplicaciones de mensajería instantánea no está exento de limitaciones obstruyen las relaciones entre los miembros de un grupo y el proceso comunicativo en sí mismo, especialmente, la incomodidad que suele presentarse en los chats grupales en conversaciones largas o complejas pudiéndose generar conflictos en la emisión de mensajes confusos.

\section{PLATAFORMAS EDUCATIVAS O SISTEMAS DE GESTIÓN DEL APRENDIZAJE}

LosSistemas degestióndelaprendizaje (Learning Management System o LMS) son soluciones de software que gestionan la administración, el seguimiento y la generación de informes de programas educativos como cursos u objetos virtuales de aprendizaje (Albarracín-Villamizar et al., 2020). Sirve como un aula virtual donde los docentes pueden interactuar con sus estudiantes y realizar actividades de aprendizaje en línea, la cual cuenta con un conjunto de herramientas como cuestionario, talleres, foros, blogs, wikis, base de datos de actividades, encuestas, chat, glosarios y evaluaciones por pares, entre otras (Rizales-Semprum et al., 2019).

Este tipo de sistemas han sido la solución fundamental durante este tiempo que existen protocolos de aislamiento y distanciamiento social. Dentro de su ventajas (FernándezPampillón, 2009), se pueden mencionar que Ios LMS como aulas virtuales no dependen de la distancia ni la ubicación, por lo que proporciona la flexibilidad y accesibilidad tanto a docentes como a estudiantes. Solo se necesita conectividad a Internet y un dispositivo. 
Garantiza la efectividad en el aprendizaje en línea ya que proporciona herramientas y funciones como distribuir materiales de aprendizaje complementarios a sus estudiantes en el curso, publicar recordatorios, entregar tareas y administrar evaluaciones. Ofrece integración de contenido multimedia como videos, enlaces, uso compartido de documentos, conferencias en vivo entre otras. Asimismo, sirve como un repositorio de materiales de aprendizaje relevantes para el curso. Los hace fácilmente accesibles, copiados y descargados por cualquier persona inscrita en el curso. Monitorear el progreso y el desempeño de los estudiantes mediante el análisis e informes sobre la efectividad de los cursos en línea: seguimiento de registros, calificaciones, envíos y participación en el curso. Asimismo, recopila archivos y documentos importantes compartidos por los estudiantes en la plataforma.

\section{MÉTODO}

\section{DISEÑO}

Para responder a las preguntas de investigación y lograr el objetivo del estudio, se adoptó una metodología cuantitativa a nivel descriptivo.

\section{POBLACIÓN Y MUESTRA}

Este estudio toma como objeto de estudio instituciones de educación superior ubicadas en Colombia. La investigación se refiere a 5 universidades que otorgan títulos de Comunicación Social y afines. Para el desarrollo de la investigación se aplicó un cuestionario en línea dirigido a los estudiantes matriculados en estos programas para el primer semestre de 2021. De acuerdo con cifras del MEN en 2018 se matricularon en estos programas 10.252 nuevos estudiantes (el $60.5 \%$ son mujeres, y el $39.5 \%$ hombres). Los cuestionarios recopilados y válidos para el análisis fueron 606 formularios. Se excluyeron de los encuestados a los estudiantes de primer semestre académico de la carrera.

\section{PROCEDIMIENTOS PARA LA RECOLECCIÓN DE LA INFORMACIÓN}

Se aplicó una prueba piloto para establecer la claridad del instrumento y medir el tiempo de diligenciamiento del mismo. Posteriormente, se procedió con implementación de la encuesta en las cinco universidades estando siempre un miembro del equipo investigador en línea con los estudiantes para resolver inquietudes que se les pudieran presentar.

\section{INSTRUMENTO DE INVESTIGACIÓN}

El cuestionario constaba de cuatro secciones. La sección uno incluyó datos demográficos, es decir, género, edad, universidad, denominación del programa, y semestre que cursa. Las siguientes tres secciones se referían a:

- Dimensión 1: Ventajas y desventajas del uso de WhatsApp como canal de comunicación interactiva.

- Dimensión 2: Ventajas y desventajas del campus virtual institucional (TEAMS, Moodle, Google Classroom)

- Dimensión 3: Competencias TIC que poseen los docentes en el uso de WhatsApp y el campus virtual institucional para el desarrollo de sus clases durante el periodo de aislamiento social causado por el COVID-19.

Se pidió a los encuestados que evaluaran el uso de las redes sociales mediante una escala Likert de 5 puntos (donde $1=$ Totalmente de acuerdo, 2 = De acuerdo, $3=$ Indiferente, $4=\mathrm{En}$ desacuerdo, $5=$ Totalmente desacuerdo).

El cuestionario se puso a prueba para 30 estudiantes para garantizar una redacción adecuada, y garantizar una validez de contenido. Como resultado de esta prueba piloto, se realizaron algunos cambios, en la redacción 
para que su contenido fuera entendido por los estudiantes.

Se siguió la metodología para la encuesta en línea propuesta en la literatura (Díaz, 2012).

La fiabilidad de las medidas se aseguró utilizando el alfa de Cronbach, que estaba por encima de 0.70 para todos los ítems (Oviedo \& Campos, 2005), es decir, 0.91 para la Dimensión 1 Ventajas y desventajas del uso de WhatsApp como canal de comunicación interactiva; 0.89 para la Dimensión 2: Ventajas y desventajas del campus virtual institucional; y 0.90 para la Dimensión 3: Competencias TIC que poseen los docentes en el uso de WhatsApp y el campus virtual institucional para el desarrollo de sus clases durante el periodo de aislamiento social causado por el COVID-19;

\section{ANÁLISIS DE LOS DATOS}

Para el análisis de la encuesta, se adoptaron frecuencias y porcentajes para analizar el perfil de los encuestados. Se adoptaron estadísticas descriptivas, es decir, media y desviación estándar, para analizar los ítems de la escala. EI cuestionario se analizó utilizando SPSS versión 25.

\section{RESULTADOS}

\section{CARACTERÍSTICAS DEMOGRÁFICAS DE LOS ENCUESTADOS}

Por medio de la Tabla 1 se referencian los aspectos característicos del perfil demográfico de los estudiantes encuestados. Se resalta el predominio del género femenino en los estudiantes encuestados, dónde el $89.0 \%$ tienen edades que oscilan entre 15 y 22 años. En cuanto a la ubicación geográfica de la oferta de los programas en los que están matriculados los encuestados, se determinó que el 53.0\% de ellos se ubican en el Gran Santander (Norte de Santander y Santander) mientras que el porcentaje restante en la Costa Caribe de Colombia. En lo concerniente a la denominación académica, se puede evidenciar que en dos terceras partes se ha acompañado de un enfoque adicional a la formación del Comunicador Social. Finalmente, se logró conformar una muestra que tiene cobertura del $89 \%$ de los semestres.

Tabla 1. Perfil demográfico de los informantes

\begin{tabular}{|c|c|c|c|}
\hline Variable & Nivel de respuesta & Frecuencia & Porcentaje \\
\hline \multirow{4}{*}{ Género } & Femenino & 428 & $70.6 \%$ \\
\hline & Masculino & 162 & $26.7 \%$ \\
\hline & LGTBI & 16 & $2.6 \%$ \\
\hline & Total & 606 & $100.0 \%$ \\
\hline \multirow{5}{*}{ Rango de edad } & De 15 a 18 años & 130 & $21.5 \%$ \\
\hline & De 19 a 22 años & 409 & $67.5 \%$ \\
\hline & De 23 a 26 años & 56 & $9.2 \%$ \\
\hline & Mayor a 26 años & 11 & $1.8 \%$ \\
\hline & Total & 606 & $100.0 \%$ \\
\hline \multirow{6}{*}{$\begin{array}{l}\text { Ubicación } \\
\text { geográfica de } \\
\text { la Institución } \\
\text { Educativa }\end{array}$} & Barranquilla & 119 & $19.6 \%$ \\
\hline & Cúcuta & 194 & $32.0 \%$ \\
\hline & Bucaramanga & 127 & $21.0 \%$ \\
\hline & Montería & 57 & $9.4 \%$ \\
\hline & Cartagena & 109 & $18.0 \%$ \\
\hline & Total & 606 & $100.0 \%$ \\
\hline
\end{tabular}




\begin{tabular}{|l|c|c|c|}
\hline \multirow{4}{*}{$\begin{array}{l}\text { Denominación } \\
\text { académica del } \\
\text { programa }\end{array}$} & Comunicación Social & 261 & $43.0 \%$ \\
\cline { 2 - 4 } & $\begin{array}{c}\text { Comunicación Social y } \\
\text { Periodismo }\end{array}$ & 224 & $37.0 \%$ \\
\cline { 2 - 4 } & $\begin{array}{c}\text { Comunicación Social y Medios } \\
\text { Digitales }\end{array}$ & 121 & $20.0 \%$ \\
\cline { 2 - 4 } & Total & $\mathbf{6 0 6}$ & $\mathbf{1 0 0 . 0 \%}$ \\
\cline { 2 - 4 } & Segundo & 60 & $9.9 \%$ \\
\cline { 2 - 4 } & Tercero & 93 & $15.3 \%$ \\
\cline { 2 - 4 } $\begin{array}{l}\text { Semestre } \\
\text { que cursa } \\
\text { actualmente }\end{array}$ & Cuarto & 51 & $8.5 \%$ \\
\cline { 2 - 4 } & Quinto & 151 & $24.9 \%$ \\
\cline { 2 - 4 } & Sexto & 52 & $8.6 \%$ \\
\cline { 2 - 4 } & Séptimo & $\mathbf{3 1 3}$ & $18.6 \%$ \\
\cline { 2 - 4 } & Octavo & $\mathbf{6 0 6}$ & $\mathbf{3 9}$ \\
\cline { 2 - 4 } & Noveno & $\mathbf{1 0 0 . 0 \%}$ \\
\hline
\end{tabular}

Fuente: Elaboración propia

\section{ANÁLISIS COMPARATIVO DE RECURSOS UTILIZADOS}

Por simplicidad en la interpretación de los resultados, los investigadores han decidido reducir la escala valorativa de cinco a tres niveles, quedando así: las opciones Totalmente de acuerdo y De acuerdo se agrupan en el nivel de Percepción Positiva, la opción de Indiferente se mantiene constante, pero las opciones En desacuerdo y Totalmente en desacuerdo se agrupan en el nivel de Percepción Negativa.

Figura 1. Comparativo de los dos recursos analizados en cuanto a las dimensiones analizadas

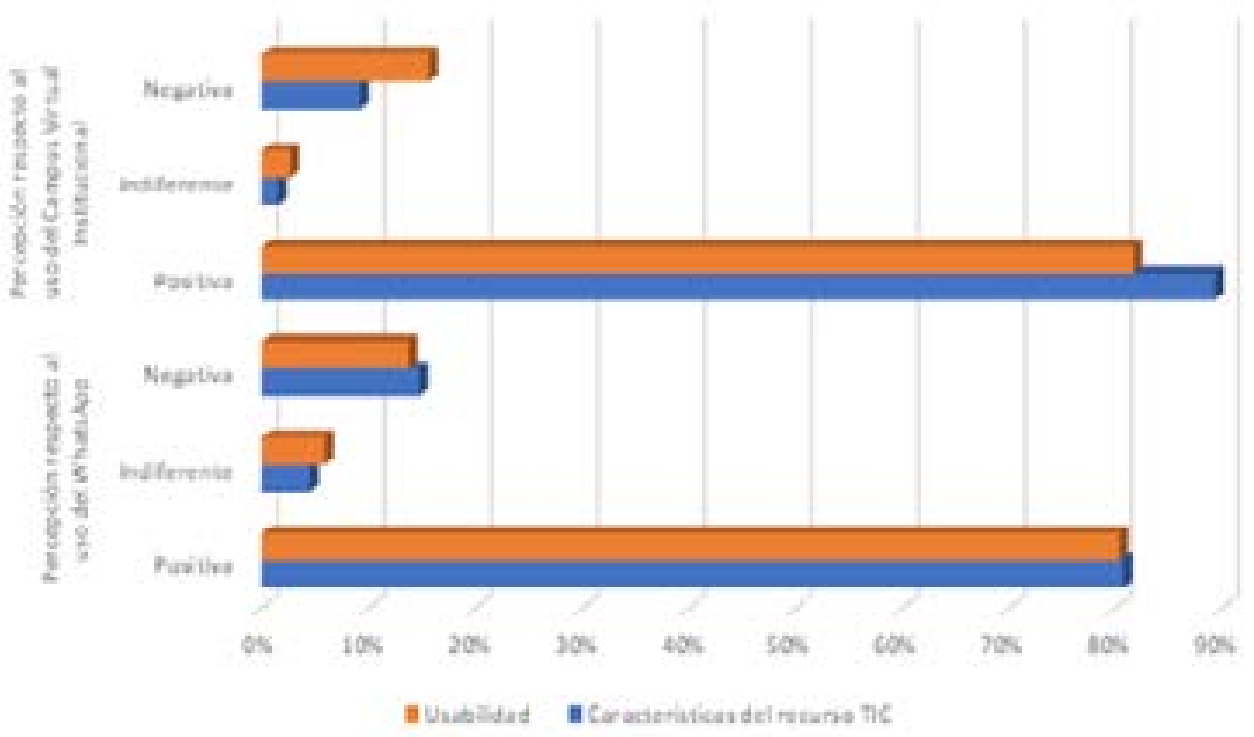


Por medio de la Figura 1 se puede observar que independientemente del recurso tecnológico utilizado por los docentes como medio de comunicación para el desarrollo de las clases en tiempos del Covid-19, el porcentaje de favorabilidad en las dos dimensiones de análisis es de al menos el $80 \%$ entre los encuestados.

Se resalta que, en opinión de los estudiantes encuestados, las plataformas institucionales en las que se han incorporado diversos recursos diseñados para el desarrollo de actividades grupales ofrecen mayores y mejores características que favorecen la comunicación interactiva entre los actores del proceso educativo, resaltando como aplicaciones más importantes Microsoft Teams (64.6\%), seguido de lejos por Google Classroom (13.5\%), plataformas libres de Moodle (10.2\%), Google Meet (7.8\%) y plataformas institucionales con impronta institucional $(3.9 \%)$; resultados que superan de forma ligera la dimensión de usabilidad de la misma, puesto que esta dimensión resulta cuestionada por los estudiantes (percepción negativa del $16 \%$ aproximadamente).

Al analizar los resultados derivados del uso del WhatsApp como recurso de apoyo para facilitar la comunicación entre los docentes y los estudiantes en el desarrollo de los cursos durante el aislamiento social por causa del Covid-19, se evidencia como opinión generalizada que este recurso presta un servicio, pero es claro que su campo de acción no es precisamente, el uso educativo.

Es importante observar en detalle cada una de las subdimensiones, puesto que se puede empezar a identificar similitudes y diferencias entre los dos recursos tecnológicos utilizados y que son el motivo de este estudio. En las Figuras 2 y 3 se pueden observar y comparar los porcentajes de favorabilidad en opinión de los estudiantes encuestados.

Subdimensión: Características del nuevo medio como canal de comunicación interactiva que utilizan los docentes con fines académicos para desarrollar sus clases durante este período de aislamiento social.

Como se observa por medio de la información mostrada en la Figura 2, se puede identificar que los porcentajes de percepción positiva respecto al uso del campus virtual institucional superan en todos los casos el $83.0 \%$ de las opiniones, aspecto que no ocurre con la aplicación de WhatsApp donde se dificulta un buen número de actividades tales como la navegación entre blogs de diversos autores, la divulgación de instrucciones de forma segmentada o la realización de búsqueda de antecedentes en diversas bases de datos, en opinión de al menos uno de cada cinco estudiantes. 
Figura 2. Comparativo de los dos recursos analizados en cuanto a sus características para la comunicación interactiva entre los docentes y sus estudiantes

\begin{tabular}{|c|c|c|c|c|c|c|}
\hline \multirow{2}{*}{$\begin{array}{l}\text { Ventajas y des ventajas del uso del recurso tecnológico } \\
\text { analizado como medio de comunicación interactiva }\end{array}$} & \multicolumn{3}{|c|}{ Percepción respecto al uso del Whats App } & \multicolumn{3}{|c|}{$\begin{array}{l}\text { Percepción respecto al uso del Campus } \\
\text { Virtual Institucional }\end{array}$} \\
\hline & Positiva & Indiferente & Negativa & Positiva & Indiferente & Negativa \\
\hline 1.1.1. Digitalización & $84.6 \%$ & $4.0 \%$ & $11.3 \%$ & $94.2 \%$ & $0.7 \%$ & $5.1 \%$ \\
\hline Compartir material de consulta bibliográfica & $82.3 \%$ & $4.5 \%$ & $13.2 \%$ & $93.8 \%$ & $0.5 \%$ & $5.7 \%$ \\
\hline Compartir material de organización de la clase & $88.2 \%$ & $3.0 \%$ & $8.8 \%$ & $95.5 \%$ & $0.5 \%$ & $4.0 \%$ \\
\hline Generar contenido colectivo & $83.4 \%$ & $4.6 \%$ & $12.0 \%$ & $93.4 \%$ & $1.0 \%$ & $5.6 \%$ \\
\hline 1.1.2. Reticularidad & $77.6 \%$ & $4.9 \%$ & $17.5 \%$ & $89.2 \%$ & $1.4 \%$ & $9.4 \%$ \\
\hline Divulgar instrucciones en forma segmentada & $71.5 \%$ & $5.3 \%$ & $23.2 \%$ & $87.2 \%$ & $1.2 \%$ & $11.6 \%$ \\
\hline Propiciar el trabajo colaborativo en pequeños grupos & $82.9 \%$ & $4.3 \%$ & $12.8 \%$ & $90.7 \%$ & $1.3 \%$ & $8.0 \%$ \\
\hline Propiciar el trabajo colaborativo entre todo el grupo de clase & $78.3 \%$ & $5.1 \%$ & $16.6 \%$ & $89.6 \%$ & $1.8 \%$ & $8.6 \%$ \\
\hline 1.1.3. Hipertextualidad & $73.8 \%$ & $5.9 \%$ & $20.2 \%$ & $86.6 \%$ & $2.2 \%$ & $11.2 \%$ \\
\hline Propiciar la navegación en Blog de autores & $70.2 \%$ & $6.8 \%$ & $23.0 \%$ & $84.6 \%$ & $2.6 \%$ & $12.8 \%$ \\
\hline Propiciar la navegación en bases de datos especializadas & $72.4 \%$ & $5.9 \%$ & $21.7 \%$ & $88.4 \%$ & $1.7 \%$ & $9.9 \%$ \\
\hline Propiciar la navegación en prensa digital & $78.9 \%$ & $5.1 \%$ & $16.0 \%$ & $86.8 \%$ & $2.3 \%$ & $10.9 \%$ \\
\hline 1.1.4. Multimedialidad & $81.7 \%$ & $4.6 \%$ & $13.6 \%$ & $83.6 \%$ & $2.4 \%$ & $14.0 \%$ \\
\hline Facilitar la creación de contenido multimedia & $80.0 \%$ & $4.6 \%$ & $15.4 \%$ & $85.3 \%$ & $2.5 \%$ & $12.2 \%$ \\
\hline Facilitar la navegación en cibermedios & $81.9 \%$ & $4.8 \%$ & $13.3 \%$ & $87.2 \%$ & $1.8 \%$ & $11.0 \%$ \\
\hline Facilitar la navegación en redes sociales & $83.3 \%$ & $4.5 \%$ & $12.2 \%$ & $78.3 \%$ & $3.0 \%$ & $18.7 \%$ \\
\hline 1.1.5. Interactividad & $85.9 \%$ & $3.0 \%$ & $11.1 \%$ & $92.9 \%$ & $0.7 \%$ & $6.4 \%$ \\
\hline Comunicarse con varias personas de manera simultánea & $90.4 \%$ & $1.7 \%$ & $7.9 \%$ & $88.7 \%$ & $1.2 \%$ & $10.1 \%$ \\
\hline Comaprtir información & $92.4 \%$ & $1.5 \%$ & $6.1 \%$ & $96.0 \%$ & $0.2 \%$ & $3.8 \%$ \\
\hline Planificar el desarrollo de la asignatura & $75.0 \%$ & $5.8 \%$ & $19.2 \%$ & $94.0 \%$ & $0.7 \%$ & $5.3 \%$ \\
\hline
\end{tabular}

Se determinaron similitudes en cuanto a la digitalización de información (el compartir material o la generación de contenido colectivo, principalmente) o la interactividad entre usuarios con el fin de compartir información o comunicación con grupos de personas en tiempo

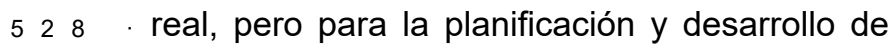
las asignaturas, las plataformas al servicio de los campus virtuales institucionales funcionan de forma más eficiente.

Se pudo identificar como principal debilidad en las plataformas de los campus virtuales institucionales, la carencia de características multimediales principalmente en la articulación con redes sociales, la creación de contenido multimedia o la existencia de vínculos de acceso a otros cibermedios.

Subdimensión: Usabilidad del recurso como canal de comunicación interactiva que utilizan los docentes con fines académicos para desarrollar sus clases durante este período de aislamiento social.

A partir de la información que se muestra por medio de la Figura 3 y analizando cada una de las características de análisis respecto a la postura positiva que manifestaron los estudiantes encuestados, se tiene que:
La aplicación WhatsApp se caracteriza por su velocidad en el intercambio de información, superando la velocidad de respuesta de cualquier de las aplicaciones implementadas en los diversos campus virtuales de las instituciones.

En cuanto a la facilidad de uso, la aplicación WhatsApp es muy axequible a cualquier persona, pero tiene limitaciones en sus complementos. En este último aspecto, las aplicaciones utilizadas en las plataformas institucionales, ofrecen amplia diversidad de herramientas que complementan la experiencia.

Al indagar sobre la periodicidad de actualización de la herramienta en uso, en opinión de los encuestados las aplicaciones incorporadas en las plataformas institucionales poseen mejor percepción que la aplicación WhatsApp, puesto que estas aplicaciones facilitan la personalización de contenidos y la incorporación de nuevas herramientas al mismo; aspectos que se ven limitados en la aplicación.

Con respecto a los procesos de retroalimentación y acompañamiento en el proceso de enseñanza, se puede identificar que los estudiantes consideran que con WhatsApp es más dinámico el proceso de clarificación de dudas y de intercambio de opiniones entre los integrantes 
del curso; pero al momento de realizar la interacción individual del docente con cada estudiante, las aplicaciones incorporadas en las diversas plataformas institucionales son más eficaces.

Cuando se indagó sobre el nivel de satisfacción que poseen los estudiantes encuestados con cada una de estas dos opciones de trabajo, se pudo identificar que WhatsApp domina el escenario cuando de controlar los contactos se trata, pero cuando el objetivo es controlar la información o adicionar complementos que apoyen los procesos de enseñanza-aprendizaje, se pudo determinar que los estudiantes consideran que las plataformas instituciones son mejores, puesto que le permite al docente incorporar recursos tales como wikis, podcast, chat, meeting, entre muchos otros.

Figura 3. Comparativo de los dos recursos analizados en cuanto a la usabilidad para la comunicación interactiva entre los docentes y sus estudiantes

\begin{tabular}{|c|c|c|c|c|c|c|}
\hline \multirow{2}{*}{$\begin{array}{l}\text { Ventajas y des ventajas del uso del recurso tecnológico } \\
\text { analizado como medio de comunicación interactiva }\end{array}$} & \multicolumn{3}{|c|}{ Percepción respecto al uso del Whats App } & \multicolumn{3}{|c|}{$\begin{array}{c}\text { Percepción respecto al uso del Campus } \\
\text { Virtual Institucional }\end{array}$} \\
\hline & Positiva & Indiferente & Negativa & Positiva & Indiferente & Negativa \\
\hline 1.2.1.Velocidad de respuesta & $79.6 \%$ & $5.7 \%$ & $14.7 \%$ & $\mathbf{7 4 . 4 \%}$ & $3.1 \%$ & $22.5 \%$ \\
\hline Es una aplicación veloz para el intercambio de información & $89.3 \%$ & $3.0 \%$ & $7.7 \%$ & $76.2 \%$ & $1.5 \%$ & $22.3 \%$ \\
\hline La App tiene una velocidad de respuesta que genera confianza & $84.1 \%$ & $4.6 \%$ & $11.3 \%$ & $74.9 \%$ & $2.6 \%$ & $22.5 \%$ \\
\hline Reduce el tiempo de entrega de las actividades asignadas & $65.4 \%$ & $9.4 \%$ & $25.2 \%$ & $72.1 \%$ & $5.1 \%$ & $22.8 \%$ \\
\hline \begin{tabular}{|l|} 
1.2.2.Facilidad de uso \\
\end{tabular} & $84.3 \%$ & $4.0 \%$ & $11.6 \%$ & $\mathbf{7 8 . 0} \%$ & $1.5 \%$ & $20.5 \%$ \\
\hline En sólo 2 o 3 clic obtiene la información que necesita & $82.7 \%$ & $4.3 \%$ & $13.0 \%$ & $76.1 \%$ & $2.0 \%$ & $21.9 \%$ \\
\hline Es fácil de utilizar por cualquier tipo de público & $90.3 \%$ & $2.5 \%$ & $7.2 \%$ & $73.2 \%$ & $0.8 \%$ & $26.0 \%$ \\
\hline Utilizan las diferentes herramientas de la aplicación & $80.0 \%$ & $5.3 \%$ & $14.7 \%$ & $84.7 \%$ & $1.7 \%$ & $13.6 \%$ \\
\hline 1.2.3. Periodicidad de actualización & $74.2 \%$ & $9.7 \%$ & $16.1 \%$ & $84.8 \%$ & $3.8 \%$ & $11.4 \%$ \\
\hline \begin{tabular}{|l} 
Utilizan la última actualización de la aplicación \\
\end{tabular} & $81.6 \%$ & $8.3 \%$ & $10.1 \%$ & $89.7 \%$ & $4.8 \%$ & $5.5 \%$ \\
\hline Personalizan sus contenidos permanentemente & $70.3 \%$ & $10.9 \%$ & $18.8 \%$ & $82.6 \%$ & $3.6 \%$ & $13.8 \%$ \\
\hline Incoporan nuevas herramientas a los contenidos que produce & $70.8 \%$ & $9.9 \%$ & $19.3 \%$ & $82.0 \%$ & $3.1 \%$ & $14.9 \%$ \\
\hline 1.2.4. Capacidad de res puesta (Retroalimentación) & $84.3 \%$ & $4.2 \%$ & $11.6 \%$ & $86.6 \%$ & $1.6 \%$ & $11.8 \%$ \\
\hline Permite aclarar dudas, evitando malos entendidos & $86.8 \%$ & $3.5 \%$ & $9.7 \%$ & $85.9 \%$ & $1.7 \%$ & $12.4 \%$ \\
\hline Contribuye al intercambio de opiniones entre compañeros & $86.4 \%$ & $4.0 \%$ & $9.6 \%$ & $86.6 \%$ & $1.7 \%$ & $11.7 \%$ \\
\hline $\begin{array}{l}\text { Facilita la retroalimentación individual del proceso de } \\
\text { aprendizaje }\end{array}$ & $79.6 \%$ & $5.0 \%$ & $15.4 \%$ & $87.3 \%$ & $1.3 \%$ & $11.4 \%$ \\
\hline 1.2.5. Nivel de satisfacción & $79.3 \%$ & $6.0 \%$ & $14.7 \%$ & $84.5 \%$ & $3.3 \%$ & $12.2 \%$ \\
\hline Brinda herramientas para el control de sus contactos & $82.0 \%$ & $5.8 \%$ & $12.2 \%$ & $81.7 \%$ & $4.1 \%$ & $14.2 \%$ \\
\hline Brinda herramientas para el control de su información & $78.3 \%$ & $5.8 \%$ & $15.9 \%$ & $88.8 \%$ & $3.3 \%$ & $7.9 \%$ \\
\hline $\begin{array}{l}\text { Los profesores complementan sus contenidos con otros } \\
\text { recursos comunicativos (sticker, mensajes de voz, emogi, gif, } \\
\text { wikis, podcast, chat, meeting) }\end{array}$ & $77.5 \%$ & $6.4 \%$ & $16.1 \%$ & $83.1 \%$ & $2.5 \%$ & $14.4 \%$ \\
\hline
\end{tabular}

\section{ANÁLISIS DE LAS COMPETENCIAS DOCENTES}

En la última sección del instrumento se incorporaron 27 ítems (distribuidos equitativamente en cada tipo de competencia) en los que cada estudiante debía evaluar el desempeño de sus docentes en tres aspectos: comunicación, uso de la tecnología y habilidades pedagógicas. Se determinó la puntuación promedio en cada tipo de competencia junto con otros estadísticos descriptivos (ver Figura 4). 
Figura 4. Estadísticos descriptivos de las competencias docentes en opinión de los estudiantes encuestados

\begin{tabular}{|c|c|c|c|c|c|c|c|c|c|}
\hline \multirow[b]{2}{*}{ Variable } & \multirow[b]{2}{*}{$\mathbf{N}$} & \multirow[b]{2}{*}{ Mínimo } & \multirow[b]{2}{*}{ Máximo } & \multirow[b]{2}{*}{ Media } & \multirow{2}{*}{$\begin{array}{c}\text { Desv. } \\
\text { Desviación }\end{array}$} & \multicolumn{2}{|c|}{ Asimetría } & \multicolumn{2}{|c|}{ Curtosis } \\
\hline & & & & & & Estadístico & $\begin{array}{l}\text { Desv. } \\
\text { Error }\end{array}$ & Estadístico & $\begin{array}{l}\text { Desv. } \\
\text { Error }\end{array}$ \\
\hline Promedio Competencias Docentes Comunicativas & 606 & 2.22 & 5.00 & 4.4571 & 0.49581 & -0.670 & 0.103 & 0.164 & 0.206 \\
\hline Promedio Competencias Tecnológicas Docentes & 606 & 2.00 & 5.00 & 4.3720 & 0.56962 & -0.900 & 0.101 & 1.250 & 0.202 \\
\hline Promedio Competencias Pedagógicas Docentes & 606 & 2.00 & 5.00 & 4.3291 & 0.55112 & -0.625 & 0.102 & 0.354 & 0.203 \\
\hline
\end{tabular}

A partir de este proceso, se pudo determinar que, en opinión de los estudiantes encuestados, los docentes son muy competentes en la socialización de los contenidos propios de las asignaturas que orientan. Al analizar la variabilidad de las competencias comunicativas de los docentes, se observa mejor desempeño con menor desviación estándar, por lo que las opiniones son muy similares en aspectos tales como: los docentes realizan actividades en tiempo real las cuales se complementan con otras independientes, favoreciendo el fortalecimiento de grupos de trabajo o comunidades de aprendizaje, que se refuerzan con la revisión de material de apoyo en diversos tipos de formato, entre los más destacados.

Cuando se analiza el comportamiento de las competencias tecnológicas, se puede determinar que las opiniones son muy diversas reflejándose en la desviación más alta, dentro de una distribución de datos con cola a la izquierda y con alta concentración de valores alrededor de la mediana (curtosis). Este aspecto se refleja en las posturas diversas que manifiestan de los docentes respecto a la utilización de portales web especializados, el uso de material digital que contribuye a desarrollar el pensamiento crítico o la generación de combinaciones de herramientas tecnológicas diversas tendientes a mejorar sus clases. Por lo anterior, se puede inferir la diversidad presente en el funcionamiento de cada programa.

Finalmente, en lo que respecta a las competencias pedagógicas exhibidas por los docentes que han orientado los diversos cursos en los que los informantes han participado, se pudo verificar que posee la puntuación media más baja, pero con alta variabilidad, lo que se podría interpretar como que las opiniones en este conjunto de competencias están divididas, principalmente en aspectos como el uso de diversos recursos TIC antes del Covid-19 en el desarrollo de sus clases o la promoción de dichos en la solución de problemas cotidianos o el desarrollo de retroalimentación por medio de plataformas institucionales.

De acuerdo con lo afirmado por Crawford-Visbal et al. (2020) y López-García y Gutiérrez-Niño (2018), el mayor desafío al que se enfrentan los docentes es superar sus propias limitaciones, para no convertirse en un obstáculo de los jóvenes universitarios que siguen dinámica acelerada y ascendente de la apropiación tecnológica.

\section{CONCLUSIONES}

El objetivo principal de esta investigación fue reportar el comparativo entre dos recursos tecnológicos disponibles (plataformas institucionales y la red social WhatsApp) que han sido utilizados como herramienta de apoyo para el desarrollo de sus clases durante el período de aislamiento social derivado del Covid-19 en cinco instituciones de educación superior del país en las que se oferta el programa de Comunicación Social.

Frente a la retroalimentación y acompañamiento en el proceso de enseñanza, los estudiantes consideran que con WhatsApp es más dinámica la clarificación de dudas y de intercambio de opiniones entre los integrantes del curso; sin embargo, en el momento de la interacción 
individual del docente con cada estudiante, las aplicaciones incorporadas en las diversas plataformas institucionales son más eficaces. Estas proveen privacidad y bidireccionalidad en comparación con la aplicación de mensajería instantánea, en donde normalmente la comunicación se da a través de un grupo creado para la clase, prestándose para confusiones en la emisión y recepción de los mensaje, como lo señalan (Vilches \& Reche, 2019).

Otro factor relevante es la oficialidad. Mientras que en la App la comunicación es espontánea y auxiliar, las plataformas, por ser los canales adoptados por las universidades, concentran legalidad y legitimidad, especialmente durante la evaluación o la entrega de trabajos de clase.

Pese a las variadas interacciones que permite el WhatsApp, los encuestados valoran las posibilidades que les ofrecen las plataformas institucionales para complementar contenidos con otros recursos comunicativos. Esto asociado al orden que les pueden dar los docentes al ambiente virtual de aprendizaje y que sin duda en la App no se aprecia

\section{REFERENCIAS}

Albarracín, C. Z., Hernández, C. A. \& Rojas, J. P. (2020). Objeto virtual de aprendizaje para desarrollar las habilidades numéricas: una experiencia con estudiantes de educación básica. Panorama, 14(26), 111-133. https://doi.org/10.15765/pnrm. v14i26.1486

Andújar-Vaca, A. \& Cruz-Martínez, M. S. (2017). Mensajería instantánea móvil: Whatsapp y su potencial para desarrollar las destrezas orales. Comunicar, 25(50), 43-52. https://doi.org/10.3916/C502017-04
Asociación Colombiana de Facultades y Programas de Comunicación. (2020). Profesiones de la comunicación $y$ transformaciones del mundo del trabajo - Informe ejecutivo de la investigación. Afacom.

Besalú-Casademont, R., Schena, J. \& SánchezSánchez, C. (2017). Competencias más relevantes en los estudios de Comunicación Audiovisual. Las percepciones de profesionales, docentes y estudiantes. Revista Latina de Comunicación Social, 72, 1.5361.553. http://doi.org/10.4185/RLCS2017-1233

Celina, H. \& Campos, A. (2005). Aproximación al uso del coeficiente alfa de Cronbach. Revista colombiana de psiquiatría, 34(4), 572-580.

Coll, C. \& Moreneo, C. (2008). (eds.). Psicología de la educación virtual. Ediciones Morata.

Crawford-Visbal, J. L, Crawford-Tirado, L., Ortiz-Záccaro, Z. Z. \& Abalo, F. (2020). Competencias Digitales en estudiantes de Comunicación a través de cuatro universidades latinoamericanas. Education in the Knowledge Society 21, 1-20. https://doi.org/10.14201/ eks.19112

Diaz, V. (2012). Ventajas e inconvenientes de la encuesta por Internet. Papers: revista de sociologia, 97(1), 193-223. https:// papers.uab.cat/article/view/v97-n1-diaz

Espinel, G. A., Hernández, C. A. \& Prada, R. (2021). Usos y gratificaciones de las redes sociales en adolescentes de educación secundaria y media: de lo entretenido a lo educativo. Encuentros, 19(01) https://doi.org/10.15665/encuen. $\underline{\mathrm{v} 19 \mathrm{i} 01.2552}$ 
Espinel-Rubio, G. A., Hernández-Suárez, C. A. \& Rojas-Suarez, J. P. (2020). Usos, apropiaciones y nuevas prácticas comunicativas de los usuarios adolescentes de Facebook. Saber, Ciencia Y Libertad, 15(1), 280-296. https://doi.org/10.18041/2382-3240/ saber.2020v15n1.6316

Fernández-Pampillón, A. (2009). Las plataformas e-learning para la enseñanza y el aprendizaje universitario en Internet. En C. López \& M. Matesanz, Las plataformas de aprendizaje. Del mito a la realidad (pp 45-73), Madrid.

Fondevila-Gascón, J. F., Marqués-Pascual, J., Mir-Bernal, P. \& Polo-López, M. (2019): Usos del WhatsApp en el estudiante universitario español. Pros y contras. Revista Latina de Comunicación Social, 74, 308-324. https://doi.org/10.4185/ RLCS-2019-1332

García, C., López, J. C. \& Correa, L. M. (2018). Usos y competencias TIC en estudiantes de comunicación social en Norte de Santander. Nexus Comunicación, (23), 82-97. https://doi.org/10.25100/ nc.v0i23.7477

López-García, J. D., \& Gutiérrez-Niño, D. (2018). Efecto del uso de la herramienta "realidad aumentada" en el rendimiento académico de estudiantes de Educación Básica. Revista Perspectivas, 3(1), 6-12. https://doi.org/10.22463/25909215.1464

Montilla, Y. N. (2020). WhatsApp como herramienta educativa en la enseñanza aprendizaje. Revista Vinculando, 1-5. https://vinculando.org/beta/whatsappcomo-herramienta-educativa-en-laensenanza-aprendizaje.html
Prada-Núñez, R., Hernández-Suárez, C. A. \& Gamboa-Suárez, A. A. (2019). Usos y efectos de la implementación de una plataforma digital en el proceso de enseñanza de futuros docentes en matemáticas. Revista Virtual Universidad Católica del Norte, (57), 137-156. http://doi.org/10.35575/rvucn. $\underline{\mathrm{n} 57 \mathrm{a} 10}$

Prada-Núñez, R., Hernández-Suárez, C. A. \& Maldonado-Estévez, E. A. (2020). Diagnóstico del potencial de las redes sociales como recurso didáctico en el proceso de enseñanza en época de aislamiento social. Revista; Espacios, 41(42), 260-268. https://doi. org/10.48082/espacios-a20v41n42p22

Rizales-Semprum, M. J, Gómez-Valderrama, C. L. \& Hernández-Suarez, C. A. (2019). Uso de herramientas tecnológicas para la enseñanza de las ciencias en educación media diversificada de acuerdo a la modalidad de estudio a distancia. Eco Matemático, 10(2), 35-46.

Rodríguez, D. (2020). Más allá de la mensajería instantánea: WhatsApp como una herramienta de mediación y apoyo en la enseñanza de la Bibliotecología. Información, cultura y sociedad, (42), 107-126. https://doi.org/10.34096/ics. $\underline{\mathrm{i} 42.7391}$

Sánchez, J. (2009). Plataformas de enseñanza virtual para entornos educativos. PixelBit. Revista de Medios y Educación, (34), 217-233. https://recyt.fecyt.es/ index.php/pixel/article/view/61377

Suárez, B. (2018). Whatsapp: su uso educativo, ventajas y desventajas. Revista de Investigación en Educación, 16(2), 121135. https://reined.webs.uvigo.es/index. php/reined/article/view/342 
USQUID (2015). Informe per a una possible implantació d'una línia d'estudis en comunicació digital interactiva. Universitat Pompeu Fabra.

Vargas-Cubero, A. L. \& Villalobos-Torres, G. (2018). El uso de plataformas virtuales y su impacto en el proceso de aprendizaje en las asignaturas de las carreras de Criminología y Ciencias Policiales, de la Universidad Estatal a Distancia de Costa Rica. Revista Electrónica Educare, 22(1), 20-39. http://doi.org/10.15359/ ree.22-1.2

Ventura, R., Roca-Cuberes, C. \& CorralRodríguez, A. (2018). Comunicación Digital Interactiva: valoración de profesionales, docentes y estudiantes del área de la comunicación sobre las competencias académicas y los perfiles profesionales. Revista Latina de Comunicación Social, 73, 331-351. https://doi.org/10.4185/RLCS-2018$\underline{1258}$

Vilches, M. J. \& Reche, E. (2019). Limitaciones de WhatsApp para la realización de actividades colaborativas en la universidad. RIED. Revista Iberoamericana de Educación a Distancia, 22(2), 57-77. http://doi. org/10.5944/ried.22.2.23741 